Article

\title{
Fractal Dimension Differentiation between Benign and Malignant Thyroid Nodules from Ultrasonography
}

\author{
Yu Yan ${ }^{1,2}$, Wei Zhu ${ }^{2}$, Yi-yun $\mathrm{Wu}^{2, *}$ and Dong Zhang ${ }^{3, *(1)}$ \\ 1 School of Computer Science \& Engineering, Nanjing University of Science \& Technology, Nanjing 210016, \\ China; yanyucan@126.com \\ 2 Nanjing University of Chinese Medicine, Nanjing 210029, China; zhuwei0710@126.com \\ 3 Institute of Acoustics, Nanjing University, Nanjing 210093, China \\ * Correspondence: wuyi425@sina.com (Y.-y.W.); dzhang@nju.edu.cn (D.Z.)
}

Received: 1 February 2019; Accepted: 28 March 2019; Published: 10 April 2019

\begin{abstract}
Recently, there is increasing interest in identifying benign and malignant nodules by using ultrasound diagnostic technology, which should be helpful to release patients with benign thyroid nodules from suffering unnecessary needle biopsy. In this work, fractal analysis was investigated for the capability of differentiating benign from malignant thyroid nodules during ultrasonography. The B-mode images for 57 patients with suspicious thyroid nodules were captured, followed by ultrasound-guided needle aspiration. The region of interests (ROIs) were subsequently selected from ultrasound images. Then, fractal analysis was performed to extract fractal texture features, and derive the fractal dimension of ROI. The results showed that a significant difference was observed between benign and malignant thyroid nodules $(p<0.05)$, by comparing this parameter based on independent nonparametric Mann-Whitney $U$ test. It suggested that fractal dimension is a helpful feature for distinguishing thyroid nodules. In addition, fractal analysis of the B-mode image can provide a reliable reference for tissue typing in ultrasound diagnosis.
\end{abstract}

Keywords: thyroid nodules; fractal dimension; B-mode image

\section{Introduction}

Thyroid nodules are a common clinical problem, particularly in iodine-deficient areas [1,2]. Though many people have nodules, they are asymptomatic, only 3-7\% nodules harbor thyroid cancer [3,4]. However, the prevalence discovered by ultrasound (US) diagnosis increases significantly to 10-67\% [4]. The majority of nodules should be benign [5], while clinical purpose of diagnosis is to detect malignancy, which only happens to $3-7 \%$ patients with thyroid nodules. Normally, suspected nodules must be assessed with fine needle aspiration (FNA) by collecting cells for cytological examination $[6,7]$. Although currently FNA is the reference standard to identify thyroid nodules, previous studies showed FNA cytology should be indeterminate in $10-30 \%$ of thyroid nodules [8,9]. To improve diagnostic accuracy, molecular biomarkers and gene expression, combined with FNA, have been explored in recent years [10-14]. Though FNA can differentiate benign from malignant nodules, it can cause physical and psychological discomfort due to its invasive procedures. Therefore, there is an urgent need to improve ultrasound diagnostic accuracy, so as to reduce unnecessary FNA biopsies.

Although US is commonly used to identify malignant from benign nodules according to some ultrasonic characteristics, no single ultrasound feature is reported to be adequately accurate to identify malignant nodules. It has been indicated that an increased risk of thyroid cancer could be marked by numerous ultrasonographic findings, i.e., calcifications, intranodular vascularity, absent halo sign, 
irregular contour, and hypo-echogenicity $[15,16]$. However, the mentioned features are not enough to identify malignant nodules, since the echogenicities of thyroid nodules are complex [15]. In recent years, ultrasound elastography has attracted much attention in detecting malignant thyroid nodules, which is developed based on the hypothesis that a malignant lesion is stiffer than a benign one [17-20]. Although this method shows good capability for identifying malignancy in the thyroid gland, it has been indicated that this technique is unsuitable for detecting small nodule and malignant nodule in low-risk population [21-24].

Since backscattered US signals are affected by the shapes and spatial distribution of scatters in tissues, it is suggested that ultrasound images follow a random dynamic pattern related to random feature of underlying structures. Regarding the pixel intensity as the height above a plane, B-mode images are regarded as rough surfaces. Fractal analysis is a method to assess textural patterns according to the visualization of ultrasound images, which can reveal the randomness of tissues or tumors. Referring to the fractal geometry, the surface intensities of ultrasound images are regarded as fractal objects, and their characteristics can be quantitatively evaluated by using fractal dimension (FD). Fractal dimension is a non-integer number between 2-3. Previous studies have proven that FD assessment shows promising results in characterizations of the breast, liver, prostate lesions, bones, and carotid plaque [25-38]. In particular, Acharya et al. have developed a computer-aided diagnostic tool to characterize benign and malignant thyroid nodules, using some texture-based feature extraction approaches (e.g., local binary pattern, FD, Laws texture energy, and Fourier spectrum descriptor) [39]. They reported that an accuracy higher than $98 \%$ for 20 patients could be achieved by combing texture features with different classifiers (e.g., support vector machine, Sugeno Fuzzy, and Gaussian mixture model). However, the specificity and sensitivity of FD parameter for thyroid nodules had not been examined. Therefore, the present study attempts to identify malignant and benign thyroid nodules with only fractal-geometry-based textural technique.

\section{Materials and Methods}

\subsection{Imaging Colletion}

The image collection used here was conducted from 24 October to 28 November 2017. A total of 57 patients ( 15 men and 42 women with a mean age of 43 and a range between 18-75 years old) with 60 thyroid nodules were enrolled in the present work. All the patients were examined with US diagnosis and US-guided FNA, and the pathological results were used as reference standards. The patient enrollment criteria were listed as follows: (1) no radiation history on the neck; (2) no thyroid disease history; (3) US and FNA examinations taken for all enrolled patients.

\subsection{Ethics}

The study was approved by the Ethics Committee of the Jiangsu Province Hospital of TCM (Nanjing, Jiangsu, China) and informed patient consent was waived. All the approaches used in the present work were performed in accordance with approved guidelines.

\subsection{Thyroid US Examination and US-Guided FNA}

Conventional US diagnosis were carried out with Vinno 70 Doppler ultrasound system (VINNO Technology Co., Ltd., Suzhou, China) using a 6-18 MHz high-resolution linear transducer (X6-16L). The mechanical index was set to be 1.2, with a depth of $38 \mathrm{~mm}$, a probe frequency of $10 \mathrm{MHz}$, a thermal index of soft tissue of 0.8 , and a total gain between 95 and 110 . The time gain compensation was fixed at near and far fields, and the focus was set at the image center. B-mode US was performed by two experienced sonographers to select the region of interest (ROI) and measure the size of thyroid lesion based on the following protocols: (1) The patients were examined in the supine position; (2) US probe was positioned over patient's thyroid to capture B-mode image in longitudinal section, avoiding the trachea and blood vessels and minimizing the artifacts as 
much as possible; (3) after a static frozen image was obtained, an ROI was marked on the thyroid lesion with a typical dimensions between $4.5 \mathrm{~mm} \times 2 \mathrm{~mm}$ and $25 \mathrm{~mm} \times 15 \mathrm{~mm}$. The features of individual thyroid nodules were characterized according to their shape, echogenicity and calcification. Echogenicity was divided into hypo- or hyper-echoic (nodules demonstrating hypoechogenicity comparing to surrounding thyroid parenchyma). Calcification (if present) was classified into mixed calcifications, microcalcifications or macrocalcifications including eggshell calcifications. The shape was measured in the transverse direction, and identified as taller or wider. All cases were thereafter assessed with Doppler examination to evaluate the vascularization of thyroid and nodules. A qualitative assessment of thyroid vascularization, which was labeled as either absent or present, was performed in the present work.

Subsequent US-guided FNAs were applied under the ultrasonographic guidance by an interventional radiologist with a 24-gauge needle. After determining the nodule's position under US guidance, FNA samples were obtained at least quartic inside each thyroid nodule in the US scanning plane, and local anesthesia was applied routinely. The tissue specimen in the needle was placed on glass slides, and then immediately put in $95 \%$ ethanol for hematoxylin eosin (HE) staining. Histological diagnoses were made by two experienced pathologists, and the final histological result of the FNAs was classified as either benign or malignant based on standard pathological criteria.

\subsection{Fractal Analysis}

The concept of fractal dimension was first introduced by Mandelbrot to describe the fractal geometry [40,41]. Various fractal models have been developed to estimate fractal dimensions of images, including fractal Brownian motion, box counting and fractal interpolation function system $[28,29,42]$. In this study, the fractional Brownian motion model was employed to analyze thyroid nodules, which has shown to be promising in estimating the roughness of some tissue images [28,42]. The detailed processing procedure is described elsewhere [29]. In brief, the B-mode images were firstly preprocessed using basic morphological operations to remove the noise imposed on the image. The most fundamental morphological operations were dilation and erosion, which are based on shapes. Dilation process added pixels to object boundaries in an US image, while the erosion process removed pixels on the boundaries of objects. The number of pixels added or removed from the objects was dependent on the shape and size of structuring element used for image processing. Then, histogram equalization was applied to standardize the gray levels in the ultrasound images for obtaining comparable fractal texture features and good contrast.

The fractal analysis was performed to extract the features of fractal texture. The Hurst coefficient $\mathrm{H}$ for the standardized image is defined as

$$
E\left[I\left(x_{2}\right)-I\left(x_{1}\right)\right] \propto\left(x_{2}-x_{1}\right)^{H}
$$

where $x_{1}, x_{2}$ are the position for pixels 1 and 2, respectively. $I\left(x_{1}\right)$ and $I\left(x_{2}\right)$ are the intensities of the two pixels in the image.

Given an $\mathrm{M} \times \mathrm{M}$ image, $\mathrm{H}$ can be estimated as the slope:

$$
\begin{gathered}
\log (d i(k))=H \log (k)+\log (c), \quad k=1,2, \ldots, n \\
\operatorname{di}(k)=\left\{\begin{array}{l}
\sum_{x=0}^{M-1} \sum_{y=0}^{M-k-1}|I(x, y)-I(x, y+k)| /[M(M-k)] \\
+\sum_{y=0}^{M-1} \sum_{x=0}^{M-k-1}|I(x, y)-I(x+k, y)| /[M(M-k)] \\
+\sum_{x=0}^{M-k-1} \sum_{y=0}^{M-k-1}|I(x, y)-I(x+k, y+k)| /(M-k)^{2} \\
+\sum_{x=0}^{M-k-1} \sum_{y=0}^{M-k-1}|I(x, M-1-y)-I(x+k, M-1-k-y)| /(M-k)^{2}
\end{array}\right\} / 4
\end{gathered}
$$


where $c$ is the scaling constant and $I(x, y)$ is the intensity of the pixel $(x, y)$ in the image. The processing procedure is executed by the MATLAB program (Math Works, Natick, MA, USA).

Finally, the fractal dimension $(D)$ of this image can be obtained as follows:

$$
D=3-H
$$

\subsection{Statistical Analysis}

A two-tailed Mann-Whitney $U$ test was carried out to evaluate whether or not the fractal dimensions of the malignant and benign nodules were significantly different.

\section{Results}

\subsection{Charactization of Thyroid Nodules}

A total of 60 thyroid nodules were studied here, which included 23 benign (follicular adenoma, colloid nodule, and cyst) and 37 malignant nodules (papillary carcinoma). The size of nodules ranged between 4 to $35 \mathrm{~mm}$ with a mean size of $15.3 \times 10.5 \mathrm{~mm}^{2}$. Table 1 lists the ultrasonographic features of all patients. It was found that the percent of taller shapes in malignant nodules (59.5\%) was much larger than in benign nodules (21.8\%). Hypoechogenicity was also a sonographic feature of malignant nodules: The percent of hypoechogenicity of malignant nodules $(81.0 \%)$ was much higher than that of benign nodules $(52.2 \%)$. No statistical difference in nodule shape was observed between the malignant and benign nodules. The presence of calcifications of the malignant nodules $(70.2 \%)$ was much higher than of benign ones (21.7\%). However, no significant difference was observed between malignant and benign nodules with regard to the vascularity of Doppler examination.

Table 1. Features of benign/malignant thyroid nodules in US images.

\begin{tabular}{cccc}
\hline \multirow{2}{*}{ Features } & & Benign & Malignant \\
\hline \multirow{2}{*}{ Shape } & taller & 5 & 22 \\
& wider & 18 & 15 \\
\hline \multirow{2}{*}{ echogenicity } & hypoechoic & 12 & 30 \\
& hyperechoic & 11 & 7 \\
\hline \multirow{2}{*}{ Calcification } & absent & 17 & 11 \\
& present & 5 & 26 \\
\hline \multirow{2}{*}{ Vascularity } & absent & 18 & 32 \\
& present & 5 & 5 \\
\hline
\end{tabular}

A longitudinal B-mode image of the normal thyroid was shown in Figure 1 where homogeneous isoechoic structure and nearly regular well-defined margins could be found in the image. Based on the echogenicity change in B-mode ultrasonography, the pathological change of thyroid gland was determined in clinics. Moreover, thyroid nodules could be classified by boundaries of ROI and texture features in images. Figure 2 shows distinct ultrasound features and accordingly pathologic findings comparing between benign and malignant lesions. A thyroid adenoma (Figure 2a) with ultrasonographic features of hyperechoic echogenicity, no calcification, and wider shape, was benign, finally proven by FNA findings. Its pathologic result showed that the follicular cavity is filled with gelatinous material, and that a papillary structure is partially visible (Figure 2c). On the contrary, hypoechoic echogenicity, microcalcification, and taller shape demonstrated a malignant papillary thyroid carcinoma (Figure 2b), with complex branched papillaries containing fibrous vascular cores (Figure 2d). 


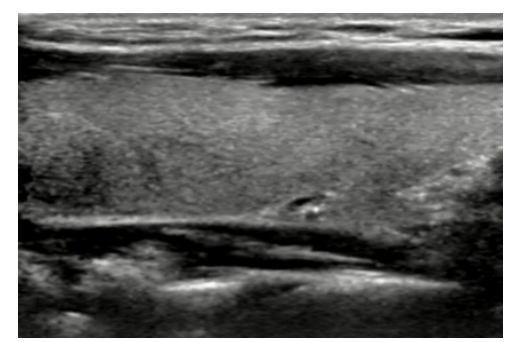

Figure 1. A longitudinal B-mode image of the normal thyroid.

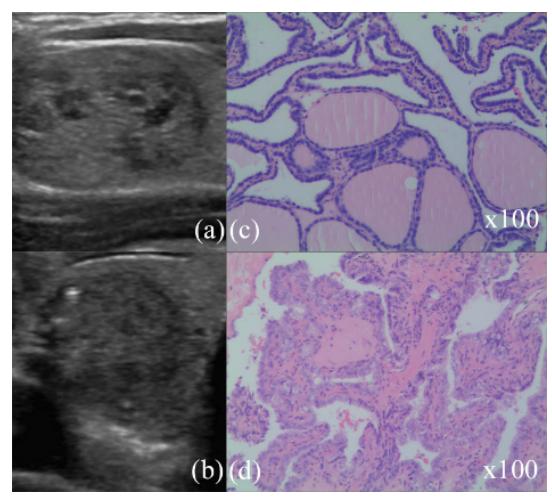

Figure 2. B-mode US images and corresponding FNAs of benign and malignant thyroid nodules: (a) B-mode image of benign thyroid nodule; (b) B-mode image of malignant thyroid nodule; (c) FNA smears of benign thyroid nodule; and (d) FNA smears of malignant thyroid nodule.

\subsection{Fractal Dimensions}

The fractal analysis can evaluate the self-similarity of all B-mode images by calculating their fractal dimensions. The process procedure for the B-mode thyroid nodule image is shown in Figure 3. A benign thyroid nodule $(9.5 \mathrm{~mm} \times 5.5 \mathrm{~mm})$ and a malignant thyroid nodule $(8.6 \mathrm{~mm} \times 8.6 \mathrm{~mm})$ were chosen to illustrate the estimation procedure. The thyroid nodule was picked up from the B-mode image for further processing. Then, morphological operations, including erosion and dilation, were applied to remove random noise superimposed on US image. The structuring element used in the morphological operation was a nonflat "ball-shaped" with radius $r=3$ and height $h=3$. Following the morphological operation, histogram equalization was operated to standardize the image and enhance its features, which was necessary for further feature extraction. After the image procession, the Hurst coefficient $\mathrm{H}$ can be calculated from the standardized images by Equation (2), where the parameter $n$ $=8$. The slope of fitting line in Figure 4 was the Hurst parameters $\mathrm{H}$ for the two nodules in Figure 2 . Finally, the fractal dimension (D) was deduced according to Equation (3).

The fractal dimensions for all 60 thyroid nodules are given in Figure 5. A two-sample independent nonparametric Mann-Whitney $U$ test was applied to evaluate whether or not the fractal dimensions of two categories were different. This test returned a parameter of $p$ for null hypothesis that two samples have equal median; a smaller $\mathrm{p}$ means the median for one sample is more different from another. A significant difference $(p<0.05)$ was observed in fractal dimensions between malignant and benign thyroid nodules.

Suspicious nodules, which are likely to be malignant, can be identified earlier before FNA by setting a certain threshold for FD. The receiver operating characteristic (ROC) curves analysis was applied using MATLAB program (Math Works). The calculated result is shown in Figure 6. The optimal threshold 2.164 was then determined by Jordan index. The area under the curve (AUC) analysis for the threshold was 0.792 , and the sensitivity and specificity were $64.9 \%$ and $86.9 \%$, respectively. 

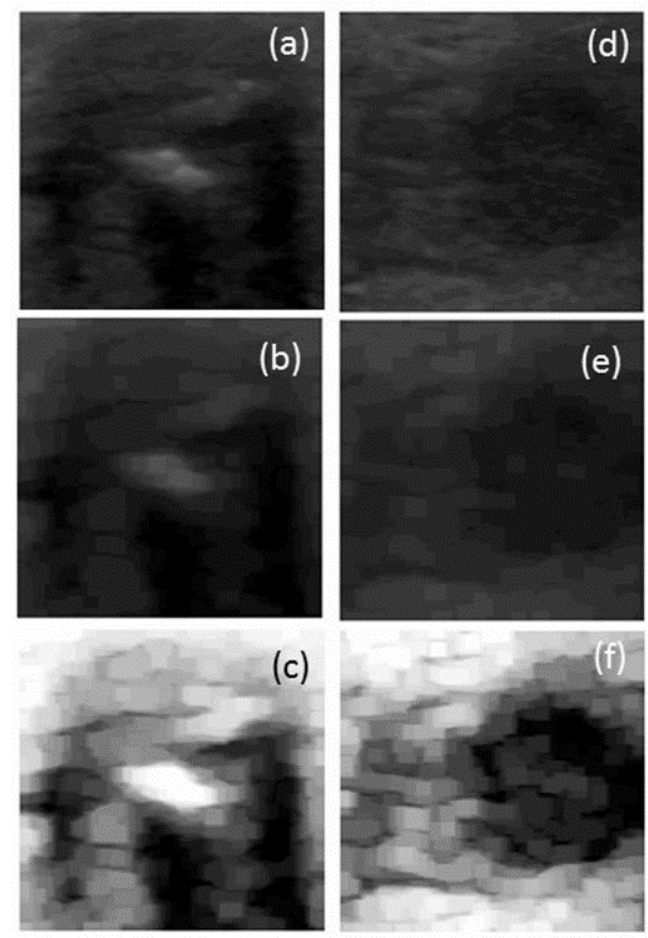

Figure 3. The processing procedure of the fractal analysis for the B-mode image. The malignant thyroid nodule: (a) Original image; (b) morphologically operated image; (c) image equalization. The benign thyroid nodule: (d) Original image; (e) morphologically operated image; (f) image equalization.

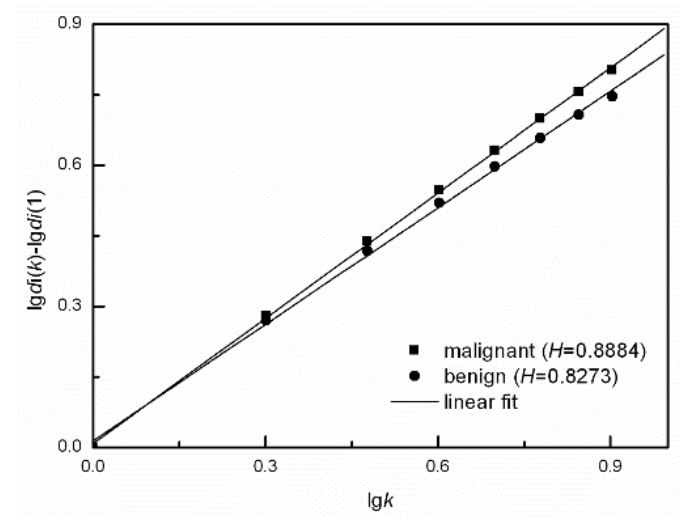

Figure 4. Fractal analysis of images in Figure 2.

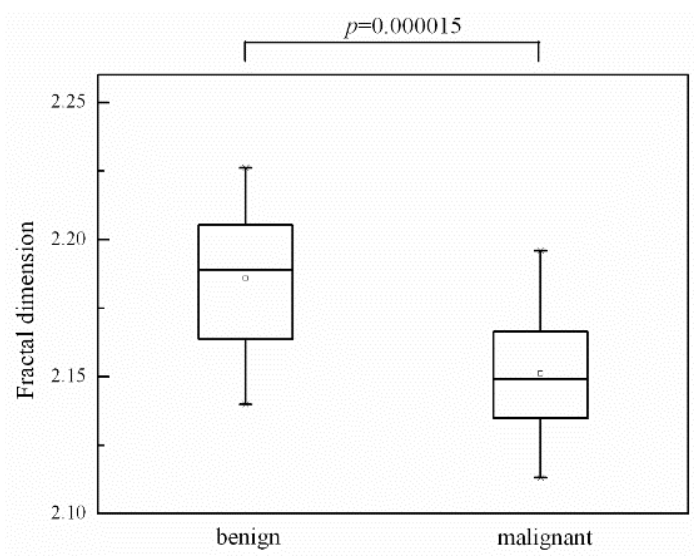

Figure 5. The fractal dimensions of benign and malignant nodules. 


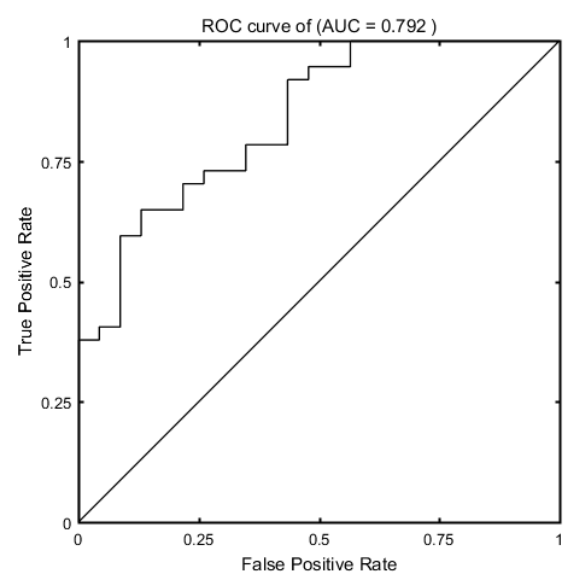

Figure 6. ROC analysis was calculated by MATLAB program and AUC was 0.792.

\section{Discussion}

The aim of the present work was to explore if the fractal analysis could be used to identify benigh nodules from malignant ones. The fractal dimensions for the two kinds of thyroid nodules were estimated based on fractal Brownian motion. The calculation results showed that the fractal dimensions of the benign nodules are generally higher than those of malignant ones (Figure 5). A similar phenomenon was observed by Chen et al. [29]. Significant difference was noticed in fractal dimension between B-mode images of the malignant and benign thyroid nodules with an independent nonparametric Mann-Whitney U test $(p<0.05)$ (Figure 5). Fractal analysis was performed to classifying breast US images and other organs and has shown promising results [27-29]. Our experimental results proved that the fractal analysis could be used as a useful tool to extract the texture properties of thyroid nodules.

The AUC value in Figure 6 was 0.792, and corresponding specificity and sensitivity were $86.9 \%$ and $64.9 \%$, respectively. Clearly, the single-parameter FD cannot be claimed as a wonderful diagnostic tool to assess thyroid nodules. Therefore, a combination of other features (e.g., texture, morphology, and so on) should be helpful for improving the classification accuracy and also be more adaptable for clinical diagnoses of different types of tissues. For instance, Acharya et al. reported that an accuracy higher than $98 \%$ was achieved for 20 patients with the combination of texture features with different classifiers [39]. More efforts will be made in the future to explore the combination of FD with other features, and with the help of multi-feature extraction methods.

In this work, the size of nodules ranged between $4.5 \times 2 \mathrm{~mm}^{2}$ to $25 \times 15 \mathrm{~mm}^{2}$, and more than $50 \%$ of them were small nodules $(<10 \mathrm{~mm})$. Compared with the conventional US diagnostic method and elastography $[20,21]$, the fractal analysis was helpful to determine whether or not a small nodule is suspicious for malignancy. Therefore, fractal dimension might be used to improve the classification performance of existing technology.

Of course, there are some limitations in the current work. First, since the ROI of thyroid nodule was determined by two senior sonographers, the results might have been affected by their subjective assessment. Thus, in future studies, computer-aided adaptive algorithms are needed to automatically select the ROI with greater objectivity. Second, all the US images studied in this work were obtained from daily diagnosis, without specific conditions set for the patients. The signal-to-noise ratio (SNR) of US images may vary between individual patients. In this case, referring to the methods introduced in a previous work [29], morphology and histogram equalization were applied to achieve noise filtering, shape simplification, and gray level standardization. However, these imaging processing methods were still too general to deal with complicated issues. Therefore, better noise reduction and US image enhancement should be explored in our future work. Meanwhile, since the size of the thyroid nodules varied in a broad range, here we only calculated the FD globally. In our future work, more efforts will be made to explore an appropriate method to estimate D locally. Moreover, there are many other 
models that exist to evaluate the FD, such as box counting, fractal interpolation function system, and area measurement $[28,42,43]$, while only the fractional Brownian motion model was employed in the present work. In the future, more assessment methods should be tested and compared to find an optimal model and parameters for a typical case, so that better performance of FD analyses can be achieved in classifying benign and malignant lesions in different organs.

\section{Conclusions}

In conclusion, a method was proposed to estimate thyroid nodules with fractal analysis, which includes the following steps: (1) Preprocess the B-mode images by using basic morphological operations in order to minimize the noise imposed on US image, (2) apply histogram equalization to standardize gray levels in the ultrasound images for obtaining comparable fractal texture features and good contrasts, (3) apply the fractal analysis to extract fractal texture features, and (4) derive fractal dimension D of the image. The results of the current work are in good agreement with FNA results. With this approach, suspicious nodules, which might be malignant, can be identified earlier before FNA by setting a certain threshold. Therefore, this method could be developed as a useful tool for US diagnosis of thyroid diseases, which should be helpful for identifying suspicious nodules so that unnecessary FNA could be avoided for benign nodules.

Author Contributions: Y.Y., Y.-y.W., and D.Z. conceived and designed the study; Y.-y.W. acquired the data. Y.Y. and W.Z. analyzed the data; Y.-y.W. and D.Z. wrote the paper. All the authors contributed to this paper.

Funding: This work was partially supported by the Jiangsu Province Key Research \& Development Plan (Grant No. BE2018703), the National Natural Science Foundation of China (Grant No. 11674173), and the QingLan Project.

Conflicts of Interest: The authors declare no conflicts of interest.

\section{References}

1. Mclver, B. Evaluation of the thyroid nodule. Oral Onclol. 2013, 49, 645-653. [CrossRef]

2. Gharib, H. Changing trends in thyroid practice: Understanding nodular thyroid disease. Endocr. Pract. 2004, 10, 31-39. [CrossRef]

3. Hegedis, L. Clinical practice. The thyroid nodules. N. Engl. J. Med. 2004, 351, 1764-1771. [CrossRef]

4. Guth, S.; Theune, U.; Aberle, J.; Galach, A.; Bamberger, C.M. Very high prevalence of thyroid nodules detected by high frequency (13 MHz) ultrasound examination. Eur. J. Clin. Investig. 2009, 39, 699-706. [CrossRef]

5. Burman, K.D.; Wartofsky, L. Thyroid Nodules. N. Engl. J. Med. 2015, 373, 2347-2356. [CrossRef]

6. Cooper, D.S.; Doherty, G.M.; Haugen, B.R.; Kloos, R.T.; Lee, S.L. Revised american thyroid association management guidelines for patients with thyroid nodules and differentiated thyroid cancer: The american thyroid association (ATA) guidelines taskforce on thyroid nodules and differentiated thyroid cancer. Thyroid 2009, 19, 1167-1214. [CrossRef]

7. Gharib, H.; Papini, E.; Paschke, R.; Duick, D.; Valcavi, R.; Hegedüs, L.; Vitti, P.; Endocrinologi, A.M. American Association of Clinical Endocrinologists, Associazione Medici Endocrinologi, and European Thyroid Association Medical guidelines for clinical practice for the diagnosis and management of thyroid nodules: Executive summary of recommendations. Endocr. Pract. 2009, 16, 468-475. [CrossRef]

8. Ohori, N.P.; Schoedel, K.E. Variability in the atypia of undetermined significance/follicular lesion of undetermined significance diagnosis in the Bethesda System for Reporting Thyroid Cytopathology: Sources and recommendations. Acta Cytol. 2011, 55, 492-498. [CrossRef]

9. Baloch, Z.W.; LiVolsi, V.A.; Asa, S.L.; Rosai, J.; Merino, M.J.; Randolph, G.; Vielh, P.; DeMay, R.M.; Sidawy, M.K.; et al. Diagnostic terminology and morphologic criteria for cytologic diagnosis of thyroid lesions: A synopsis of the National Cancer Institute Thyroid Fine-Needle Aspiration State of the Science Conference. Diagn. Cytopathol. 2008, 36, 425-437. [CrossRef]

10. Lin, J.D.; Chao, T.C.; Huang, B.Y.; Chen, S.T.; Chang, H.Y.; Hsueh, C. Thyroid cancer in the thyroid nodules evaluated by ultrasonography and fine-needle aspiration cytology. Thyroid 2005, 15, 708-717. [CrossRef] 
11. Xing, M.; Haugen, R.R.; Schlumberger, M. Progress in molecular-based management of differentiated thyroid cancer. Lancet 2013, 381, 1058-1069. [CrossRef]

12. Finley, D.J.; Zhu, B.; Barden, C.B.; Fahey, T.J., III. Discrimination of benign and malignant thyroid nodules by molecular profiling. Ann. Surg. 2004, 240, 425-437. [CrossRef] [PubMed]

13. Zheng, B.; Liu, J.; Gu, J.; Lu, Y.; Zhang, W. A three-gene panel that distinguishes benign from malignant thyroid nodules. Int. J. Cancer 2015, 136, 1646-1654. [CrossRef]

14. Hong, Y.R.; Liu, X.M.; Li, Z.Y.; Zhang, X.F.; Chen, M.F.; Luo, Z.Y. Real-time Ultrasound Elastography in the Differential Diagnosis of Benign and Malignant Thyroid Nodules. J. Ultrasound Med. 2009, 28, 861-867. [CrossRef] [PubMed]

15. Frates, M.C.; Benson, C.B.; Charboneau, J.W.; Cibas, E.S.; Clark, O.H.; Coleman, B.G.; Cronan, J.J.; Doubilet, P.M.; Evans, D.B.; Goellner, J.R.; et al. Management of thyroid nodules detected at US: Society of Radiologists in Ultrasound consensus conference statement. Ultrasound Q. 2006, 22, 231-238. [CrossRef]

16. Desser, T.S.; Kamaya, A. Ultrasound of thyroid nodules. Neuroimaging Clin. N. Am. 2008, 18, 463-478. [CrossRef] [PubMed]

17. Garra, B.S. Imaging and estimation of tissue elasticity by ultrasound. Ultrasound Q. 2007, 23, $255-268$. [CrossRef]

18. Ueno, E.; Ito, A. Diagnosis of breast cancer by elasticity imaging. Eizo Joho Med. 2004, 36, 2-6.

19. Asteria, C.; Giovanardi, A.; Pizzocaro, A.; Cozzaglio, L.; Morabito, A.; Somalvico, F.; Zoppo, A. US-elastography in the differential diagnosis of benign and malignant thyroid nodules. Thyroid 2008, 18, 523-531. [CrossRef]

20. Dighe, M.; Bae, U.; Richardson, M.; Dubinsky, T.; Minoshima, S.; Kim, Y. Differential diagnosis of thyroid nodules with US elastography using carotid artery pulsation. Radiology 2008, 248, 662-669. [CrossRef]

21. Rago, T.; Santini, F.; Scutari, M.; Pinchera, A.; Vitti, P. Elastography: New developments in ultrasound for predicting malignancy in thyroid nodules. J. Clin. Endocrinol. Metab. 2007, 92, 2917-2922. [CrossRef] [PubMed]

22. Shao, J.; Shen, Y.; Lü, J.; Wang, J. Ultrasound scoring in combination with ultrasound elastography for differentiating benign and malignant thyroid nodules. Clin. Endocrinol. (Oxf.) 2015, 83, 254-260. [CrossRef]

23. Friedrich-Rust, M.; Ong, M.F.; Martens, S.; Sarrazin, C. Performance of transient elastography for the staging of liver fibrosis: A meta-analysis. Gastroenterology 2008, 134, 960-974. [CrossRef] [PubMed]

24. Vidal-Casariego, A.; López-González, L.; Jiménez-Pérez, A.; Ballesteros-Pomar, M.D.; Kyriakos, G.; Urioste-Fondo, A.; Álvarez-San Martín, R.; Cano-Rodríguez, I.; Jiménez-García de la Marina, J.M. Accuracy of ultrasound elastography in the diagnosis of thyroid cancer in a low-risk population. Exp. Clin. Endocrinol. Diabetes 2012, 120, 635-638. [CrossRef] [PubMed]

25. Asvestas, P.; Golemati, S.; Matsopoulos, G.K.; Nikita, K.S.; Nicolaides, A.N. Fractal dimension estimation of carotid atherosclerotic plaques from B-mode ultrasound: A pilot study. Ultrasound Med. Biol. 2002, 28, 1129-1136. [CrossRef]

26. Wu, C.M.; Chen, Y.C.; Hsieh, K.S. Texture Features for Classification of Ultrasonic Liver Images. IEEE Trans. Med. Imaging 1992, 11, 141-152. [PubMed]

27. Uniyal, N.; Eskandari, H.; Abolmaesumi, P.; Sojoudi, S.; Gordon, P.; Warren, L.; Rohling, R.N.; Salcudean, S.E.; Moradi, M. Ultrasound RF time series for classification of breast lesions. IEEE Trans. Med. Imaging 2015, 34, 652-661. [CrossRef] [PubMed]

28. Moradi, M.; Abolmaesumi, P.; Isotalo, P.A.; Siemens, D.R.; Sauerbrei, E.E.; Mousavi, P. Detection of Prostate Cancer from RF Ultrasound Echo Signals Using Fractal Analysis. In Proceedings of the 2006 International Conference of the IEEE Engineering in Medicine and Biology Society, New York, NY, USA, 30 August-3 September 2006; Volume 1, pp. 2400-2403.

29. Chen, D.R.; Chang, R.F.; Chen, C.J.; Ho, M.F.; Kuo, S.J.; Chen, S.T.; Hung, S.J.; Moonm, W.K. Classification of breast ultrasound images using fractal feature. Clin. Imaging 2005, 29, 235-245. [CrossRef]

30. Fiz, J.A.; Monte-Moreno, E.; Andreo, F.; Auteri, S.J.; Sanz-Santos, J.; Serra, P.; Bonet, G.; Castellà, E.; Manzano, J.R. Fractal dimension analysis of malignant and benign endobronchial ultrasound nodes. BMC Med. Imaging 2014, 14, 22. [CrossRef]

31. Veltri, M.; Ferrari, M.; Balleri, P. Correlation of radiographic fractal analysis with implant insertion torque in a rabbit trabecular bone model. Int. J. Oral Maxillofac. Implants 2011, 26, 108-114. 
32. Ilhan, B.; Guneri, P.; Saracoglu, A.; Koca, H.; Boyacioglu, H. A comparison of fractal dimension values of peri-implant bone and healthy contralateral side using panoramic radiographs. J. Oral Maxillofac. Radiol. 2015, 3, 1-6. [CrossRef]

33. Zhou, R.; Luo, Y.K.; FensterJohn, A.; Spence, D.; Ding, M.Y. Fractal dimension based carotid plaque characterization from three-dimensional ultrasound images. Med. Biol. Eng. Comput. 2018, 57, $135-146$. [CrossRef] [PubMed]

34. Lee, W.L.; Chen, Y.C.; Hsieh, K.S. Ultrasonic liver tissues classification by fractal feature vector based on M-band wavelet transform. IEEE Trans. Med. Imaging 2003, 22, 382-392. [CrossRef] [PubMed]

35. Bikou, O.; Delides, A.; Drougou, A.; Nonni, A.; Patsouris, E.; Pavlakis, K. Fractal Dimension as a Diagnostic Tool of Complex Endometrial Hyperplasia and Well-differentiated Endometrioid Carcinoma. In Vivo 2016, 30, 681-690. [PubMed]

36. Maipas, S.; Nonni, A.; Politi, E.; Sarlanis, H.; Kavantzas, N.G. The Goodness-of-fit of the fractal dimension as a diagnostic factor in breast cancer. Cureus 2018, 10, e3630. [CrossRef]

37. Miwa, K.; Inubushi, M.; Wagatsuma, K. FDG uptake heterogeneity evaluated by fractal analysis improves the differential diagnosis of pulmonary nodules. Eur. J. Radiol. 2014, 83, 715-719. [CrossRef] [PubMed]

38. Lee, L.H.; Tambasco, M.; Otsuka, S. Digital differentiation of non-small cell carcinomas of the lung by the fractal dimension of their epithelial architecture. Micron 2014, 67, 125-131. [CrossRef] [PubMed]

39. Acharya, U.R.; Sree, S.V.; Krishnan, M.M.R.; Molinari, F.; Garberoglio, R.; Suri, J.S. Non-invasive automated 3D thyroid lesion classification in ultrasound: A class of ThyroScanTM systems. Ultrasonics 2012, 52, 508-520. [CrossRef]

40. Mandelbrot, B.B. How long is the coast of Britain? Statistical self-similarity and fractional dimension. Science 1967, 156, 636-638. [CrossRef]

41. Mandelbrot, B.B. Fractal Geometry of Nature; W H Freeman \& Co.: New York, NY, USA, 1983.

42. Fortin, C.; Kumaresan, R.; Ohley, W.; Hoefer, S. Fractal dimension in the analysis of medical images. IEEE Eng. Med. Biol. 1992, 11, 65-71. [CrossRef]

43. Lopes, R.; Betrouni, N. Fractal and multifractal analysis: A review. Med. Image Anal. 2009, 13, $634-649$. [CrossRef] [PubMed] 